\title{
Service robots for citizens of the future ${ }^{1}$
}

\author{
Carme Torras \\ Institut de Robòtica i Informàtica Industrial (CSIC-UPC), Barcelona \\ http://www.iri.upc.edu/research/perception \\ http://www.iri.upc.edu/people/torras
}

\begin{abstract}
Robots are no longer confined to factories, but they are progressively spreading to urban, social and assistive domains. In order to become handy co-workers and helpful assistants, they must be endowed with quite different abilities than their industrial ancestors. Research on service robots aims to make them intrinsically safe to people, easy to teach by non-experts, able to manipulate not only rigid but also deformable objects, and highly adaptable to non-predefined and dynamic environments. Robots worldwide will share object and environmental models, their acquired knowledge and experiences through global databases and, together with the internet of things, will strongly change the citizens' way of life in so-called smart cities. This raises a number of social and ethical issues that are now being debated not only within the Robotics community but by society at large.
\end{abstract}

\section{From industrial to service robotics}

In the early twenty-first century, the growing need for labor in the healthcare and service sectors - partly motivated by the ageing of population in the more developed countries- has widened the range of robot applications. In the coming years we will see robots attending elderly and disabled people, performing household tasks, acting as support teachers, assistants in shopping malls, receptionists, guides at trade-fairs and museums, and even as nannies and playmates. Not only will robots find increased application in human-centered domains such as healthcare, education and entertainment, but also in service areas like city logistics, cleaning and recycling, surveillance and environmental monitoring. Moreover, robots will also increase their range of activities in factories by working not just in production lines, but also collaborating with human operators as co-workers.

In Europe the ambitious SPARC program ${ }^{2}$ has been launched to cope with the research needs arising in this scenario. This is a contractual partnership of the European Commission and euRobotics AISBL, an association of companies and academia, to facilitate the growth and empowerment of the robotics industry and value chain, from research through to production. With $€ 700 \mathrm{M}$ in funding from the Horizon2020 program for 2014-20, and triple that amount from European industry, SPARC is the largest civilian robotics innovation program in the world. It is structured in eight application areas: manufacturing, healthcare, home care, agriculture, security, environment, transport and entertainment.

This move of robotics towards de service sector is in line with the upsurge of smart city technologies. Applications as diverse as garbage collection and recycling, surveillance 
and security, logistics and elderly assistance, require both ambient intelligence and autonomous robots. Ambitious projects in this direction are underway, such as the development of a web for robots ${ }^{3}$, where they would share data and procedures, i.e., maps of visited buildings, acquired manipulation skills, and other learned expertise, in a common hardware-independent format. This web will be connected to the internet of things, from which robots could get object models and instructions of use for all kinds of commercial products.

\section{Challenges and research approaches}

Service robots working in human environments ${ }^{4,5}$ have considerably different requirements than the industrial robots included in production lines. Since they cannot be caged, their safeness to people must be intrinsically guaranteed. In many cases there won't be expert programmers around and, for example, robot assistants should be easy to be taught by non-experts. Deformable objects such as clothing, upholstery and cables pervade human environments and robots should be able to perceive and manipulate them; this is much harder than dealing with the usual rigid workpieces in factories, because flexible shapes have a much higher dimensional state space than the six variables of position and orientation. The high accuracy required in many industrial operations can often be dispensed in this context, but in return robot performance needs to be robust in front of noisy perceptions and inaccurate actions. And, of course, service robots must be highly adaptable to non-predefined and dynamic environments.

Underlying these requirements, there is a common trait: it is impossible to program service robots by anticipating all possible circumstances in which they will have to operate, thus some form of learning is needed, although the particular technique applied will vary largely from the low-level skills (e.g., function approximation to fit trajectories) to the high-level competences (e.g., semantic labeling and symbolic reasoning), as we will explain in the following subsections.

Several research groups are conducting projects on service robotics at universities and research centers worldwide. At our institute (IRI, CSIC-UPC), two European projects addressing the aforementioned core competences in this area have been completed: URUS and PACO-PLUS, aimed at developing robots to work as urban guides and cooking assistants, respectively. Other related European projects in which we were involved are GARNICS and IntellAct, which deal with the perception and manipulation skills needed by a robot gardener and a robot performing maintenance tasks. In particular, at IRI's Perception and Manipulation group, we do research at the frontier of Robotics and AI, integrating computer vision, machine learning and planning to address several of the requirements sketched above. Hence, some of our works will be described below to illustrate the research challenges posed by service robots as well as promising ways to tackle them.

\subsection{Easy programming}

If non-experts are to instruct robots to perform tasks, very natural ways of doing so must be provided. Learning from demonstration ${ }^{6}$ has emerged as a powerful approach by means of which robots can acquire the desired skills by just being shown a demonstration from a human. Initial research relied on vision for teaching object pickand-place sequences, whereas now the focus is on accomplishing force-based dynamic 
tasks. Indeed forces play an important role in many skills that service robots should have, such as opening doors, pulling drawers, assembling things, and cutting slices of some foods, to name a few.

We have proposed a learning framework ${ }^{7}$, where teacher demonstrations are encoded in a Hidden Markov Model (HMM), and robot execution is implemented through a modified version of Gaussian Mixture Regression that uses the temporal information from the HMM. Such information is needed when tackling tasks in which force perceptions may be ambiguous, e.g., taking out a piece through a hole, where the same force may be sensed in the point where trajectories from different origins cross. Experimental results with our framework have demonstrated that the robot is able to learn and reproduce two tasks of this nature, namely dispensing pills and pouring drinks, with a performance comparable to the teacher's one.

Learning more elaborate skills (e.g., those involving complex dynamics) just from demonstrations may prove very time consuming or even impossible. Hence, it has been suggested that starting from a demonstration to bootstrap behaviour, the robot should explore slightly modified versions of the acquired skill so as to improve its performance through reinforcement learning $(\mathrm{RL})^{8}$. The difficulty is to scale $\mathrm{RL}$ to the highdimensional continuous motion spaces of robot manipulators or, even more generally, humanoid robots. To surmount this difficulty, different ways to parameterize movement primitives have been proposed, coupled with efficient policy learning procedures ${ }^{9}$, as well as strategies to reduce the dimensionality of the parameter space on which exploration needs to be carried out ${ }^{10}$. Recently, probabilistic movement primitives ${ }^{11}$ have been put forward to capture the essentials of several demonstrations, and again ways to reduce the dimensionality of the search space have been developed ${ }^{12}$.

So far we have described research on learning approaches to transfer skills from humans to robots. A step further is to teach robots not just to reproduce skills on their own, but to accomplish tasks cooperatively with humans. In a joint work with the Istituto Italiano di Tecnologia, we have proposed a framework to transfer impedance-based behaviours to a torque-controlled robot through kinaesthetic teaching ${ }^{13}$. The collaborative assembly of a wooden table is used as test-bed (see Fig. 1). The perception system consists of vision markers in the table legs and forces sensed at the robot end-effector. In the demonstration stage, the teacher makes the robot follow compliantly the guidance of the user to place the table board in a comfortable position to perform the assembly and, as soon as the user starts screwing a leg, the teacher firmly holds the robot so that the board remains stiff. The demonstrations are encoded as a task-parameterized statistical dynamical system and stiffness estimation is formulated as a convex optimization process, thus guaranteeing optimal stiffness gain matrices. The results show that the framework successfully modifies the robot impedance along task execution to facilitate collaboration, by triggering stiff and compliant behaviours in an on-line manner to adapt to the user's actions. Note that when the user erroneously tries to screw a leg upside down or in the middle of the table, the robot behaves compliantly. 

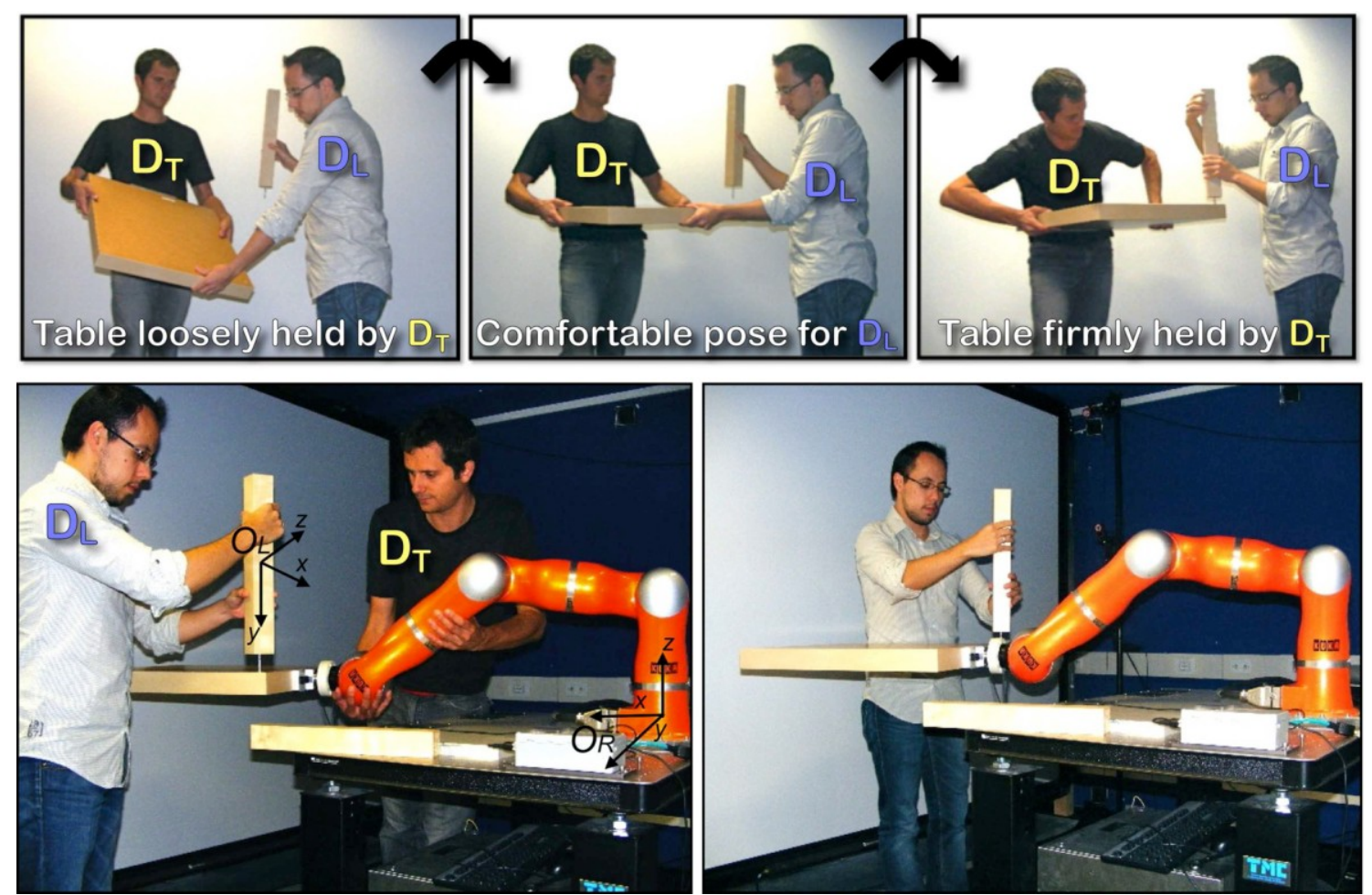

Figure 1. Easy robot programming by demonstration. Top: Two people collaborating to assemble a wooden table. Bottom-left: The teacher $\left(\mathrm{D}_{\mathrm{T}}\right)$ instructs the robot to hold the board stiff only while the human operator $\left(D_{L}\right)$ is screwing a leg. Bottom-right: The robot stops behaving compliantly when the human operator starts screwing a leg. (Adapted from Ref. 13.)

\subsection{Safety}

Service robots often have to operate near humans or even in physical contact with them as in the collaborative tasks mentioned above. Therefore, safeness to people is a sine qua non for such robots. In the manufacturing context, the usual approach has been to limit robot velocity as a function of distance to people, leading to very conservative speed or torque bounds to the detriment of productivity. Recently, a kinematic control strategy has been proposed ${ }^{14}$ that enforces safety (included as a hard constraint) while maintaining the maximum level of productivity of the robot.

The European project SAPHARI ${ }^{15}$ aims to go a step further and address safety in close contact collaborative situations by relying on human-friendly hardware design and interaction control strategies that would enable robots to track, understand and predict human motions in real-time, and to react accordingly. To this end, a hierarchical architecture $^{16}$ has been devised that generates robot behaviours organized in three layers for active collaboration, co-existence, and safety. At the upper layer, an intentional contact by a human user to request collaboration should be distinguished from an accidental collision. Human-robot co-existence requires workspace monitoring and efficient collision avoidance methods based on exteroceptive sensors. Despite these controls, unavoidable physical contacts may eventually occur anywhere on the robot body, which should be reliably detected and a fast robot reaction triggered, preferably on the basis of only proprioceptive sensing. 
We devised a method to estimate external forces exerted on a manipulator during motion based exclusively on propioception ${ }^{17}$, i.e., avoiding the use of tactile or force sensors. This estimator has been used together with a friction-aware controller in an RL approach to learn safety-critical tasks ${ }^{18}$, such as helping to dress people with reduced mobility, whereby the robot needs to behave compliantly but still adhere to a reference trajectory.

Note that to program a robot to behave compliantly, a trade-off between precision and safety needs to be considered, since augmenting robot precision (usually with a high error-compensating term) will make its motion increasingly stiff and, therefore, potentially dangerous for humans in its vicinity. This trade-off is handled by using impedance or direct force controllers that rely on an inverse dynamic model of the robot, i.e., which map position, velocity and acceleration to the torques acting on the robot. However, most approaches to build such a model do not consider the possibility of having hystheresis on the friction, which is the case for robots like the Barrett WAM. For this reason, we derived an analytical model of friction in the seven robot joints, whose parameters can be automatically tuned for each particular robot (Ref. 18). This permits compliantly adhering to reference trajectories in the entire workspace.

Experimental results demonstrate that using such friction-aware controller within a RL approach based on movement primitives, the robot is consistently capable of learning simple dressing tasks, such as wrapping a scarf around the neck of a mannequin and, afterwards, of a person ${ }^{19}$ (see Fig. 2).

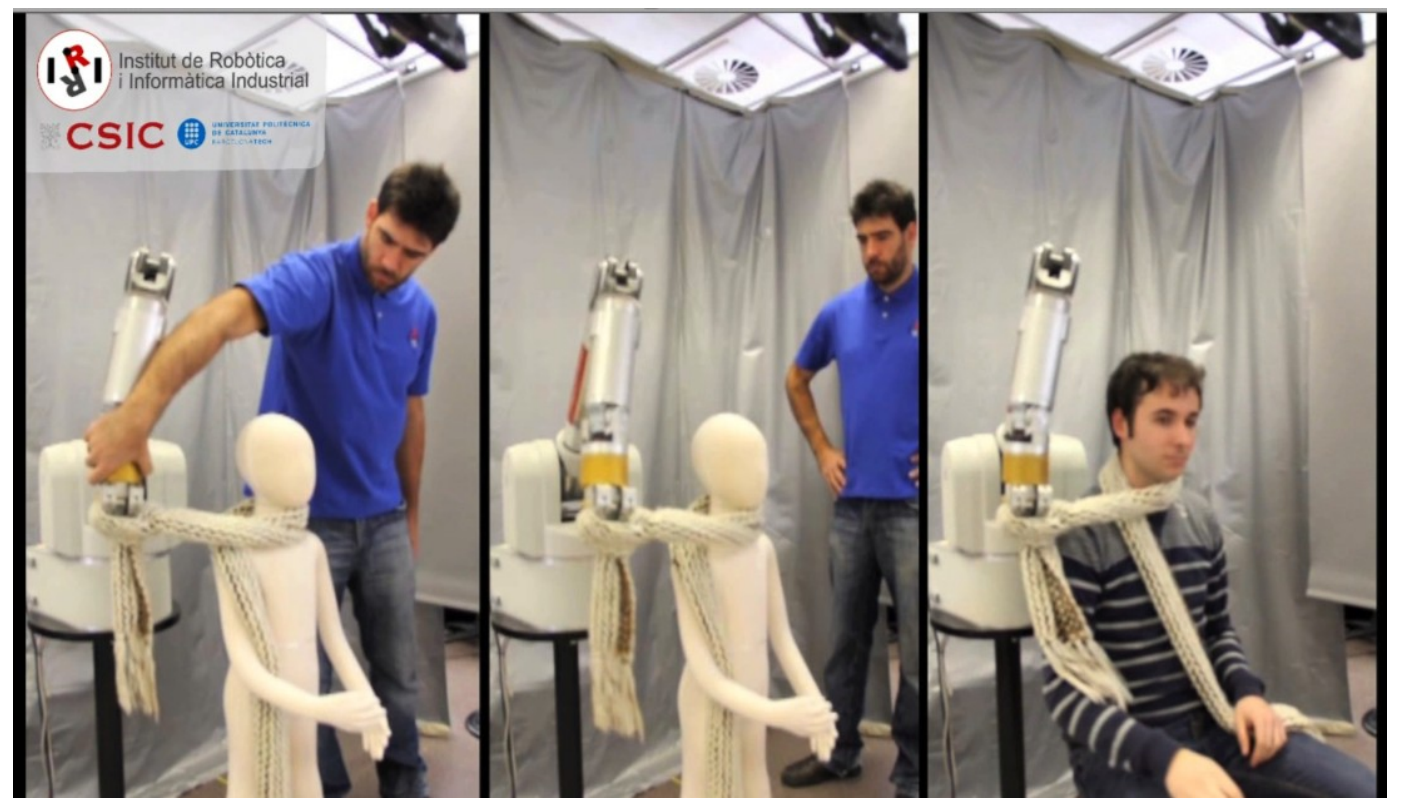

Figure 2. Using a safety-aware controller in the execution of tasks that entail physical interaction with a human. Left: The teacher demonstrates how to wrap a scarf around the neck of a mannequin by physically guiding the robot. Middle: The robot autonomously explores parameterized trajectories close to the demonstrated one within an RL approach so as to optimize performance. Right: The robot reproduces the best motion found and, as can be observed in the video in Ref. 19, despite being insistently disturbed by the user, it shows a nice compliant behaviour while at the same time persistently pursuing the task. 


\subsection{Handling deformable objects}

Whereas handling a rigid object changes only its pose, namely six parameters (three for position and three for orientation), the manipulation of a deformable object takes place in a potentially infinite-dimensional shape-state space. This huge dimensionality jump renders geometry-based perception techniques developed for rigid objects nonapplicable in this context, and calls for the use of machine learning approaches as well as applying motions to the object to aid perception. The latter has been the dominant trend in robotic handling of garments ${ }^{20,21}$, where clothes are manipulated and re-grasped until reaching a configuration that can be easily recognized with simple perception algorithms.

Since re-graspings are time consuming, we have explored the alternative approach of applying more complex computer vision and machine learning algorithms for informed (task-oriented) one-shot grasping. For instance, to hang a shirt or a coat, we should pick them up by the collar. Thus, we need to detect suitable parts of garments. To this end, we have built and made publicly available a dataset ${ }^{22}$ that includes hundreds of manually annotated RGB-D scans of clothing items, such as collars of polo shirts, hips and hemlines of pants, shirt cuffs, etc. Besides the annotated colour image, each entry in the database includes also the depth image and a template for background subtraction (see Fig. 3-left).
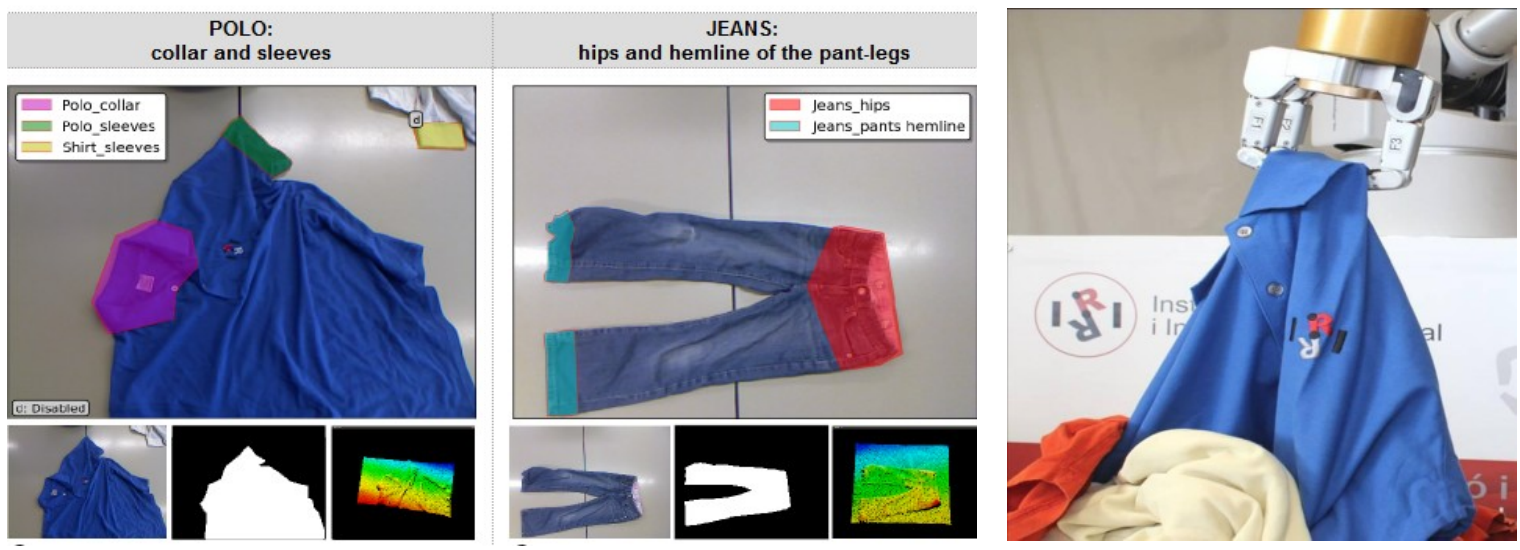

Figure 3. Perception and manipulation of deformable objects. Left: Two entries in the clothing database showing the annotated parts (top), as well as the colour and depth images, and the template for background subtraction (bottom). Right: Grasping a polo shirt by the collar for hanging.

Then, we have devised a pipeline ${ }^{23}$ where, from the colour and depth images, we extract some descriptors and, in a training phase, a dictionary of codewords is built using a Bag-of-Words approach. Next, a Support Vector Machine is used to classify garment parts. In execution, the descriptors are computed using a sliding window procedure to generate a map of part (e.g., collar) likelihood, which is then used to place boxes around the most probable part locations. Within the best box, a novel 3D descriptor ${ }^{24}$ is applied to find the most suitable grasping point on the suitable part (e.g., the collar lapel for 
hanging purposes, as shown in Fig. 3-right). Experimental results ${ }^{25}$ demonstrate the suitability of the approach for task-oriented informed grasping of clothes.

So far we have just dealt with recognition of garments and their parts. Determining the pose of clothing is a much more involved problem that requires not just classifying but determining shape, which is usually accomplished by matching points between a reference garment pose and the current, deformed one. We have developed a Deformation and Light Invariant (DaLI) descriptor ${ }^{26}$, which has been shown to outperform state-of-art descriptors, such as SIFT and DAISY.

But clothes are not the only deformable objects service robots must be able to perceive and manipulate. Upholstered furniture, cables, foods and plants are examples of other such items. The European project $\mathrm{GARNICS}^{27}$ aimed to automatically monitor large botanic experiments to determine the best treatments (watering, nutrients, sunlight) to optimize predefined aspects (growth, seedling, flowers) and to eventually guide robots to obtain relevant data from plant leaves (e.g., chlorophyll measurements with a spad meter as in Fig. 4). For this purpose, a time-of-flight camera was mounted on the robot end-effector together with the appropriate measurement tool, so as to move around the plant using a next-best-view approach to find a leaf on whose border a suitable sampling point could be reached ${ }^{28}$.

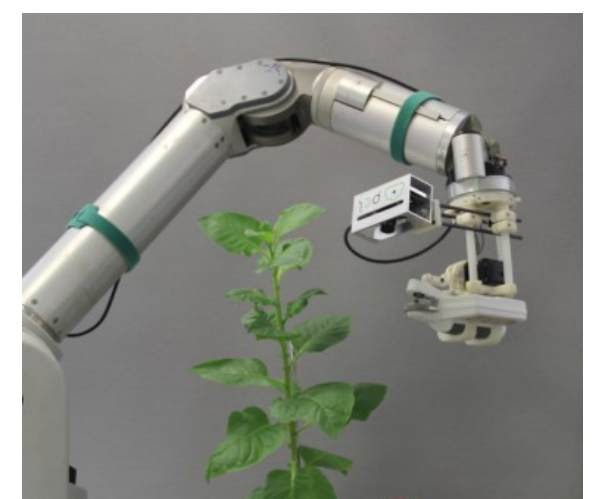

Figure 4. Setup used to take chlorophyll measures from suitable plant leaves, with the Barrett WAM arm carrying a time-of-flight camera and a spad meter. Observe that the leaf-probing task requires clearance (above and below) of a segment in the border of the chosen leaf.

\subsection{Adaptivity and learning}

Contrarily to industrial robots, which usually operate in structured, predefined settings in a repetitive way, service robots need to work in dynamic environments not specifically designed for them and often with humans around. Thus, they should have the capability to adapt to new situations and cope with unexpected events. Such adaptation takes two rather different forms depending on whether it occurs at the sensorimotor or cognitive levels ${ }^{29}$. Sensorimotor adaptation consists in building relevant associations between stimuli and responses, while cognitive learning entails constructing symbolic representations to guide decision-making.

The visuomotor mapping, relating visual input to motion commands, has been the most widely studied, it allowing robots to adapt not only to changes in the environment but also to changes in their own geometry due to damage, wear-and-tear or tool replacement. Hoffmann et al. ${ }^{30}$ provide a detailed review of robotics works tackling the acquisition of a body schema, a term taken from the neurosciences to denote the 
correlation of proprioceptive sensory information with the visible shape of the body. For articulated robots such representation is often restricted to an inverse kinematics/dynamics mapping relating the end-effector pose (position and orientation) and velocity to the robot joint angles and torques. Neural networks have been used to acquire the whole kinematics mapping from scratch, and also to encode only the deviations from the nominal kinematics embedded in the original robot controller ${ }^{31}$, resulting in a significant speed-up that permits online adaptation of the body schema, a crucial feature for service robots that need to operate for a long time without assistance. In a joint work with Karlsruhe Institute of Technology, the body schema of the humanoid robot Armar-III has been updated online to encompass a tool of variable length and pose with respect to the robot hand ${ }^{32,33}$ (see Fig. 5).

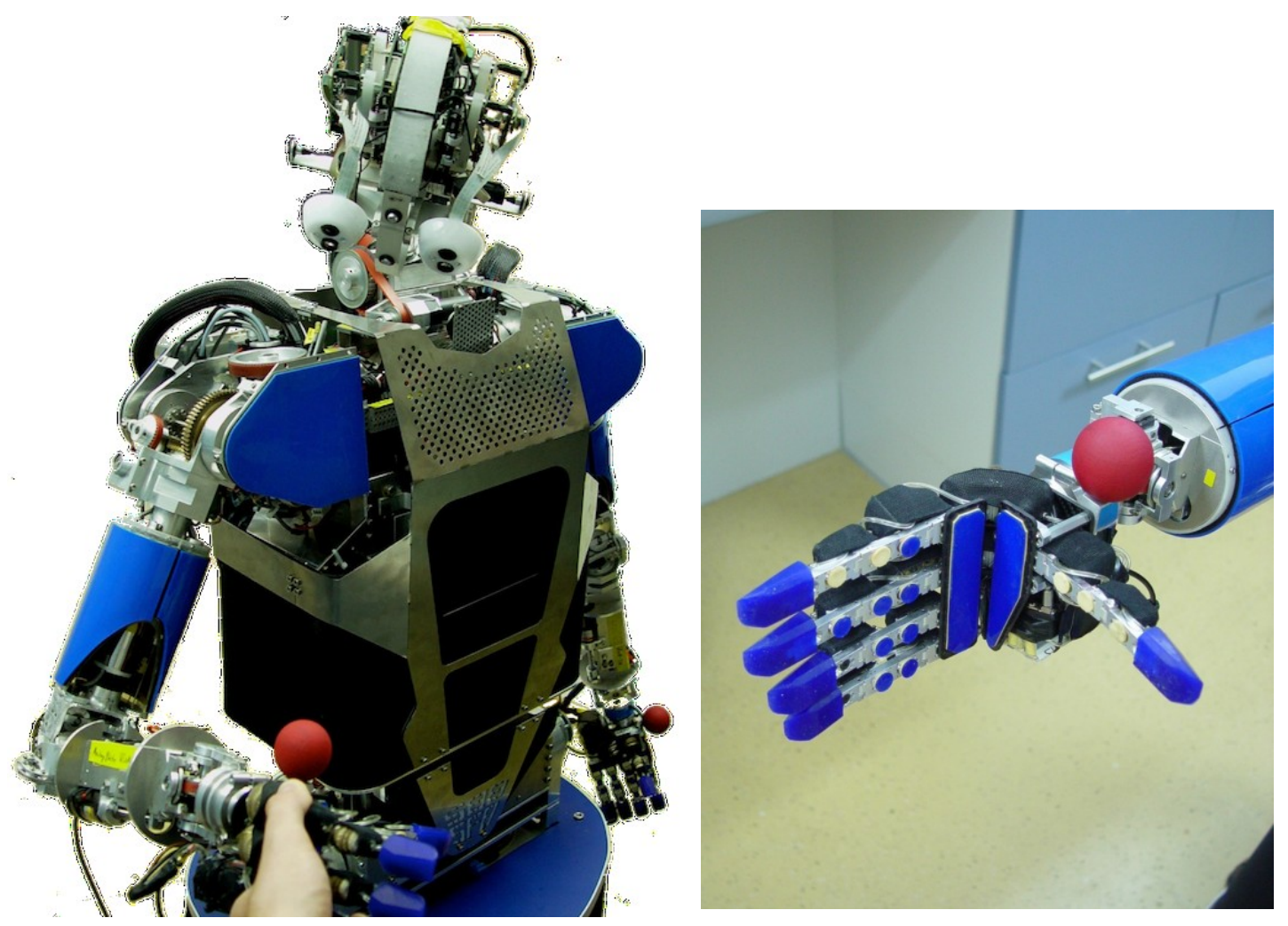

Figure 5. Left: The humanoid robot Armar-III used in the experiments to adapt the body schema to an extension of the hand with a tool. Right: Close-up of the red ball attached to the right hand marking the end of the tool. (Adapted from Ref. 33.)

For tasks involving not just a single skill but sequences of actions in non-predefined settings, planning capabilities are needed. In order to develop them, a cognitive architecture $^{34}$ should be able to progressively learn an action model from experiences and rehearse hypothetical future scenarios on that model so as to determine the best course of action. Moreover, such action model must be probabilistic to account for noise in perceptions and uncertainties in action outcomes. 
Relational reinforcement learning has been developed towards this aim. By enhancing RL with relational representations of states and actions (i.e., explicitly encoding relations in a symbolic data structure), the acquired knowledge can be generalized across states and transferred across tasks ${ }^{35}$.

Some robot actions may be irreversible leading to unrecoverable failures (e.g., damaging some robot element, breaking an object or losing a tool). On the contrary, a planner can always backtrack from a dead-end so as to try to find an alternative sequence of actions to reach the goal. Thus, prior experiences can be very helpful for safety-aware planning.

We have proposed a relational RL method that allows a robot to reason about deadends and their causes. If a plan might lead to a dead-end, the robot tries to find an alternative safe plan and, if not found, it asks a teacher whether the risky action should be executed. This method permits learning safe policies as well as minimizing unrecoverable errors during the learning process, and it has been validated on a robot learning to clear up a table ${ }^{36}$.

\section{Social and ethical implications}

The fact that service robots are intended to operate among people and often in close interaction with them raises many questions concerning their influence on the future of society and the role this technology may play in sustainability. Several roboethics initiatives and projects are underway in an attempt to address such questions in contexts as diverse as the military, labour market, legal liability, and educational fields.

In a study trying to predict how pervasive robotics may shape individual identity, we became aware of the serious methodological difficulties involved in predicting technological evolution ${ }^{37}$ : unforeseen uses of devices always crop up as in the case of cell phones; technological development cannot be studied outside its sociocultural context; and current language has severe limitations to describe the future (quoting Heidegger, it is «through technique that we perceive the sea as navigable»).

Given the difficulty of predicting how a technological society will evolve, a reasonable option is to imagine different possible future scenarios and encourage debate on the pros and cons to try to guide techno-scientific research in the most desirable direction. In a provocative speech at Arizona State University entitled "Innovation starvation"38, the renowned writer Neal Stephenson advocated that we should recur to science fiction not only to figure out innovations but, above all, to come up with coherent scenarios of how such innovations could be integrated into a society and change peoples lives. Actually, classical science fiction anticipated many of the dilemmas we are currently facing.

As an example, the scientific journal Interaction Studies devoted a special issue to discuss the influence that the use of robot nannies might have on the psychological development of children. The introductory article ${ }^{39}$ draws attention to a number of tricky issues that should be addressed. It is fascinating to realise ${ }^{40}$ that several of these issues appeared in short stories published more than half a century ago, such as "Robbie" by I. Asimov, "Nanny" by Ph. K. Dick or "I sing the body electric" by R. Bradbury. I gave my opinion on these ethical concerns in the aforementioned special issue $^{41}$, so here I will only point out that if robots are to be part of the future educational community, it is important that society as a whole forms an opinion on what type of 
robots they would accept. While there seem to be consensus on robots that increase human autonomy or amplify human capacities (e.g., the dexterity of surgeons), robots behaving as emotional substitutes raise polemics.

In an attempt to contribute to the debate, I wrote a science-fiction novel ${ }^{42}$, where I imagined how being raised by artificial nannies, learning from robot teachers and sharing work and leisure with humanoids would affect the intellectual and social habits of future generations, their feelings and relationships, enhancing or spoiling them depending on each person's point of view. The novel's leit motiv is a quotation from the philosopher R.C. Solomon: «it is the relationships that we have constructed which in turn shape us». He meant human relations with our parents, teachers and friends, but the quotation can be applied to robotic assistants and robot companions, if they are to pervade people's lives.

\section{Conclusions}

Service robots are coming to stay. They pose a whole range of challenges not only to techno-scientific research, as outlined throughout this paper, but also to the humanities, since they raise some intriguing moral dilemmas that are nowadays the subject of heated controversies.

Concerning future research, besides the expected incremental advances in human-robot interaction, safety, manipulation of soft materials, adaptivity and learning, we foresee that robot self-knowledge will be a key ingredient to significantly increase robot autonomy in the years to come. Several degrees of self-knowledge could be distinguished progressively enabling more complex functionalities. The simplest body schema consisting of a parameterized kinematic/dynamic model has already been incorporated into robots through sensorimotor learning. More elaborate self-models would include a body image precisely delimiting the robot boundaries acquired through exploratory actions, leading to the distinction between a body self and others. This would allow robots to construct a model of their own physical/cognitive abilities which, for instance, could allow them to ask for help whenever a task goes beyond their capabilities or either to explore new actions ${ }^{43,44}$ and try to acquire the required skills if no helper is around.

Developments in this direction will surely lead to more versatile, highly-performing robots, but in turn will bring about new issues and, in particular, intensify the debate on whether robots should be given more autonomy and decision making abilities, especially in critical contexts such as the military ${ }^{45}$.

In sum, service robots constitute an amazing application that is fostering robotics research, while at the same time they pose decisive questions that are triggering an exciting social and ethical debate.

\section{Acknowledgements}

The described research has been partially supported by the European projects PACOPLUS (IST-FP6-IP-027657), GARNICS (FP7-ICT-247947) and IntellAct (FP7-ICT269959), and the Spanish project PAU+ (DPI2011-27510).

\section{References}


1. This paper summarizes my presentation at the 26th Annual Conference of the Academia Europaea, Barcelona, July 2014.

\section{2. http://sparc-robotics.eu/}

3. For example, projects RoboEarth (http://roboearth.org/) and RoboHow (http://robohow.eu/).

4. C.C. Kemp, A. Edsinger and E. Torres-Jara (2007) Challenges for robot manipulation in human environments. IEEE Robotics and Automation Magazine, 14(1): 20-29.

5. C. Smith, Y. Karayiannidis, L. Nalpantidis, X. Gratal, P. Qi, D.V. Dimarogonas and D. Kragic (2012) Dual arm manipulation-A survey. Robotics and Autonomous systems, 60(10): 1340-1353.

6. A. Billard, S. Calinon, R. Dillmann and S. Schaal (2008) Robot programming by demonstration. Handbook of Robotics, chapter 59, pp. 1371-1394. Springer.

7. L. Rozo, P. Jiménez and C. Torras (2013) A robot learning from demonstration framework to perform force-based manipulation tasks. Intelligent Service Robotics, 6(1): $33-51$.

8. R.S. Sutton and A.G. Barto (1998) Reinforcement Learning: An Introduction. MIT, Cambridge. (2nd edition at https://www.dropbox.com/s/f4tnuhipchpkgoj/book2012.pdf)

9. J. Peters and S. Schaal (2008) Reinforcement learning of motor skills with policy gradients. Neural Networks, 21(4): 682-697.

10. A. Colomé and C. Torras (2014) Dimensionality reduction and motion coordination in learning trajectories with dynamic movement primitives. IEEE/RSJ Intl. Conf. on Intelligent Robots and Systems (IROS), Chicago, pp. 1414-1420.

11. A. Paraschos, G. Neumann, C. Daniel and J. Peters (2013) Probabilistic movement primitives. Advances in Neural Information Processing Systems (NIPS), Cambridge, pp. 2616-2624.

12. A. Colomé, G. Neumann, J. Peters and C. Torras (2014) Dimensionality reduction for probabilistic movement primitives. IEEE-RAS Intl. Conf. on Humanoid Robots, Madrid, Spain, pp. 794-800.

13. L. Rozo, S. Calinon, D. Caldwell, P. Jiménez and C. Torras (2013) Learning collaborative impedance-based robot behaviors. 27th Intl. Conf. of the Assoc. for the Advancement of Artificial Intelligence (AAAI-13), Bellevue, Washington, pp. 14221428 .

14. http://www.saphari.eu/ 
15. A.M. Zanchettin, N.M. Ceriani, P. Rocco, H. Ding and B. Matthias (2015) Safety in human-robot collaborative manufacturing environments: metrics and control. IEEE Trans. on Automation Science and Engineering, to appear.

16. A. De Luca and F. Flacco (2012) Integrated control for pHRI: Collision avoidance, detection, reaction and collaboration. $4^{\text {th }}$ IEEE Intl. Conf. on Biomed. Robotics and Biomechatronics (BioRob), Rome, Italy, pp. 288-295.

17. A. Colomé, D. Pardo, G. Alenyà and C. Torras (2013) External force estimation during compliant robot manipulation. IEEE Intl. Conf. on Robotics and Automation (ICRA), Karlsruhe, Germany, pp. 3535-3540.

18. A. Colomé, A. Planells and C. Torras (2015) A friction-model-based framework for reinforcement learning of robotic tasks in non-rigid environments. IEEE Intl. Conf. on Robotics and Automation (ICRA), Seattle, pp. 5649-5654.

\section{9. http://www.iri.upc.edu/groups/perception/\#ScarfTask}

20. M. Cusumano-Towner, A. Singh, S. Miller, J.F. O'Brien and P. Abbeel (2011) Bringing clothing into desired configurations with limited perception. IEEE Intl. Conf. on Robotics and Automation (ICRA), Shanghai, China, pp. 3893-3900.

21. A. Doumanoglou, A. Kargakos, T-K. Kim and S. Malassiotis (2014) Autonomous active recognition and unfolding of clothes using random decision forests and probabilistic planning. IEEE Int. Conf. on Robotics and Automation (ICRA), HongKong, pp. 987-993.

22. http://www.iri.upc.edu/groups/perception/\#clothingDataset

23. A. Ramisa, G. Alenyà, F. Moreno-Noguer and C. Torras (2012) Using depth and appearance features for informed robot grasping of highly wrinkled clothes. IEEE Intl. Conf. on Robotics and Automation (ICRA), St. Paul, Minnesota, pp. 1703-1708.

24. A. Ramisa, G. Alenyà, F. Moreno-Noguer and C. Torras (2013) FINDDD: A fast 3D descriptor to characterize textiles for robot manipulation. IEEE/RSJ Intl. Conf. on Intelligent Robots and Systems (IROS), Tokyo, Japan, pp. 824-830.

25. A. Ramisa, G. Alenyà, F. Moreno-Noguer and C. Torras (2014) Learning RGB-D descriptors of garment parts for informed robot grasping. Engineering Applications of Artificial Intelligence, 35: 246-258.

26. E. Simo-Serra, C. Torras, and F. Moreno-Noguer (2015) DaLI: deformation and light invariant descriptor. International Journal of Computer Vision, to appear.

\section{7. http://www.garnics.eu/}

28. G. Alenyà, B. Dellen, S. Foix and C. Torras (2013) Robotized plant probing: Leaf segmentation utilizing time-of-flight data. IEEE Robotics and Automation Magazine, 20(3): 50-59. 
29. C. Torras (1995) Robot adaptivity. Robotics and Autonomous Systems, 15(1): 11-23.

30. M. Hoffmann, H. Gravato, A. Hernandez, H. Sumioka, M. Lungarella and R. Pfeifer (2010) Body schema in robotics: A review. IEEE Trans. on Autonomous Mental Development, 2(4): 304-324.

31. V. Ruiz de Angulo and C. Torras (1997) Self-calibration of a space robot. IEEE Trans. on Neural Networks, 8(4): 951-963.

32. S. Ulbrich, V. Ruiz de Angulo, T. Asfour, C. Torras and R. Dillman (2009) Rapid learning of humanoid body schemas with kinematic Bezier maps. 9th IEEE Intl. Conf. on Humanoid Robots, Paris, pp. 431-438.

33. S. Ulbrich, V. Ruiz de Angulo, T. Asfour, C. Torras and R. Dillman (2012) Kinematic Bézier maps. IEEE Transactions on Systems, Man and Cybernetics: Part B, 42(4): 1215-1230.

34. D. Vernon, G. Metta, G. Sandini (2007) A survey of artificial cognitive systems: Implications for the autonomous development of mental capabilities in computational agents. IEEE Trans. on Evolutionary Computation, 11(2): 151-180.

35. D. Martínez, G. Alenyà and C. Torras (2015) Relational reinforcement learning with guided demonstrations. Artificial Intelligence Journal, to appear.

36. D. Martínez, G. Alenyà and C. Torras (2015) Safe robot execution in model-based reinforcement learning. IEEE/RSJ Intl. Conf. on Intelligent Robots and Systems (IROS), Hamburg, Germany, to appear.

37. F. Ballesté and C. Torras (2013) Effects of human-machine integration on the construction of identity. In R. Luppicini (ed.) Handbook of Research on Technoself: Identity in a Technological Society, Chapter 30, pp. 574-591, IGI Global: Hershey, EUA.

38. http://www.worldpolicy.org/journal/fall2011/innovation-starvation

39. N. Sharkey and A. Sharkey (2010) The crying shame of robot nannies. Interaction Studies: Social Behaviour and Communication in Biological and Artificial Systems, 11(2): 161-190.

40. C. Torras (2014) Social robots: A meeting point between science and fiction. Metode Science Studies Journal - Annual Review, 5: 110-115.

41. C. Torras (2010) Robbie, the pioneer robot nanny: Science fiction helps develop ethical social opinion. Interaction Studies: Social Behaviour and Communication in Biological and Artificial Systems, 11(2): 269-273.

42. C. Torras (2012) La mutación sentimental (The sentimental mutation). Editorial Milenio. 
43. A. Agostini, C. Torras and F. Wörgötter (2011) Integrating task planning and interactive learning for robots to work in human environments. Intl. Joint Conf. on Artificial Intelligence (IJCAI), Barcelona, pp. 2386-2391.

44. A. Agostini, C. Torras and F. Wörgötter (2015) Efficient interactive decisionmaking framework for robotic applications. Artificial Intelligence Journal, to appear.

45. http://www.stopkillerrobots.org/2015/07/aicall/

\begin{abstract}
About the author
Carme Torras (http://www.iri.upc.edu/people/torras) is Research Professor at the Spanish Scientific Research Council (CSIC). She received M.Sc. degrees in Mathematics and Computer Science from the Universitat de Barcelona and the University of Massachusetts, Amherst, respectively, and a Ph.D. degree in Computer Science from the Technical University of Catalonia (UPC). Prof. Torras has published five books and more than two hundred papers in the areas of robot kinematics, computer vision, geometric reasoning, machine learning and manipulation planning. She has been local project leader of several European projects in the frontier between AI and Robotics, among which the FP6 IP project "Perception, Action and COgnition through Learning of Object-Action Complexes" (PACO-PLUS), and the FP7 STREP projects "GARdeNIng with a Cognitive System" (GARNICS) and "Intelligent observation and execution of Actions and manipulations" (IntellAct). She was awarded the Narcís Monturiol Medal of the Generalitat de Catalunya in 2000, and she became ECCAI Fellow in 2007, member of Academia Europaea in 2010, and member of the Royal Academy of Sciences and Arts of Barcelona in 2013. Prof. Torras is currently Editor of the IEEE Transactions on Robotics.
\end{abstract}

